Annals of Pure and Applied Mathematics

Vol. 16, No. 1, 2018, 203-214

ISSN: 2279-087X (P), 2279-0888(online)

Published on 15 January 2018

Annals of

www.researchmathsci.org

DOI: http://dx.doi.org/10.22457/apam.v16n1a22

Pure and Applied

Mathematics

\title{
q-Analogue of Univalent Functions with Negative Coefficients
}

\section{Hamid Shamsan $^{1}$ and S. Latha ${ }^{2}$}

Department of Mathematics, Yuvaraja's College, University of Mysore

Mysore 570 005, INDIA

1hmas19771@gmail.com, ${ }^{2}$ drlatha@gmail.com

${ }^{1}$ Corresponding author

Received 6 December 2017; accepted 26 December 2017

Abstract. In this paper, we derive a necessary and sufficient condition, distortion theorem and coefficient inequalities are determined for univalent functions with negative coefficients that are $q$-starlike of order $\beta$ and $q$-convex of order $\beta$. We also establish extreme point results, some results concerning the partial sums for the function $f(z)$ belonging to the class $T_{q}^{*}(\beta)$.

Keywords: Univalent functions, Starlike, Convex, $q$-Derivative, Partial sums, Extreme points.

AMS Mathematics Subject Classification (2010): $30 \mathrm{C} 45$

\section{Introduction}

Let $S$ denote the class of functions of form

$$
f(z)=z+\sum_{n=2}^{\infty} a_{n} z^{n}
$$

Which are analytic and univalent in the open unit disk

$$
\mathrm{U}=\{z: z \in \mathrm{C} \text { and }|z|<1\} \text {, }
$$

with $S^{*}(\beta)$ and $K(\beta), 0 \leq \beta<1$, designating the subclasses of $\mathrm{S}$ consisting of functions starlike and convex of order $\beta$. We shall denote by $T$ the subclass of $\mathrm{S}$ consisting of functions that may be expressed in the form

$$
f(z)=z-\sum_{k=2}^{\infty} a_{k} z^{k}, \quad\left(a_{k} \geq 0\right)
$$

Further, let

$$
T_{q}^{*}(\beta)=S_{q}^{*}(\beta) \cap T \quad(0 \leq \beta<1)
$$

and

$$
C_{q}(\beta)=K_{q}(\beta) \cap T \quad(0 \leq \beta<1) .
$$




\section{Hamid Shamsan and S. Latha}

Jackson[2] initiated $q$-calculus and developed the concept of the $q$-integral and $q$ derivative.

For a function $f \in \mathrm{S}$ given by (1.1) and $0<q<1$, the $q$-derivative of $f$ is defined by

\section{Definition 1.1.}

$$
\partial_{q} f(z)=\left\{\begin{array}{rr}
\frac{f(z)-f(q z)}{z(1-q)}, & z \neq 0, \\
f^{\prime}(0), \quad z=0, & 0<q<1 .
\end{array}\right.
$$

Equivalently (1.5), may be written as $\partial_{q} f(z)=1+\sum_{n=2}^{\infty}[n]_{q} a_{n} z^{n-1}, \quad z \neq 0$ where

$$
[n]_{q}=\frac{1-q^{n}}{1-q} \text {. Note that as } q \rightarrow 1,[n]_{q} \rightarrow n .
$$

Making use of $\partial_{q} f$ T. M. Seoudy and M. K. Aouf [4] introduced the subclasses $S_{q}^{*}(\beta)$ and $K_{q}(\beta)$ defined by

Definition 1.2. A function $f(z) \in \mathrm{A}$ is said to be $q$-starlike of order $\beta, 0 \leq \beta<1$, if and only if $\operatorname{Re}\left\{\frac{z \partial_{q} f(z)}{f(z)}\right\}>\beta, \quad$ for all $z \in \mathrm{U}$.

We denote by $S_{q}^{*}(\beta)$ the subclass of $A$ consisting of all starlike functions of order $\beta$ in the unit disk $U$.

Definition 1.3. A function $f(z) \in \mathrm{A}$ is said to be $q$-convex of order $\beta, 0 \leq \beta<1$, if and only if $\operatorname{Re}\left\{\frac{\partial_{q}\left(z \partial_{q} f(z)\right)}{\partial_{q} f(z)}\right\}>\beta, \quad$ for all $z \in \mathrm{U}$.

We denote by $K_{q}(\beta)$ the subclass of $\mathrm{A}$ consisting of all convex functions of order $\beta$ in the unit disk $U$.

We note that $f \in K_{q}(\beta)$ if and only if $z \partial_{q} f \in S_{q}^{*}(\beta)$

$$
\text { and } \lim _{q \rightarrow 1^{-}} S_{q}^{*}(\beta)=S^{*}(\beta) \text {, and } \lim _{q \rightarrow 1^{-}} K_{q}(\beta)=K(\beta),
$$

where $S^{*}(\beta), K(\beta)$ are the classes of starlike and convex functions of order $\beta$ respectively.

\section{Main results}

Theorem 2.1. A function $f(z)=z+\sum_{k=2}^{\infty} a_{k} z^{k}$ is in $S_{q}^{*}(\beta)$, if

$$
\sum_{k=2}^{\infty}\left([k]_{q}-\beta\right)\left|a_{k}\right| \leq 1-\beta .
$$


Hamid Shamsan and S. Latha

Proof: It suffices to show that the values for $\frac{z \partial_{q} f(z)}{f(z)}$ lie in a circle centered at $\omega=1$ whose radius is $1-\beta$.

$$
\begin{aligned}
& \left|\frac{z \partial_{q} f(z)}{f(z)}-1\right|=\left|\frac{z \partial_{q} f(z)-f(z)}{f(z)}\right|=\left|\frac{\sum_{k=2}^{\infty}\left([k]_{q}-1\right) a_{k} z^{k}}{z+\sum_{k=2}^{\infty} a_{k} z^{k}}\right| \\
& \leq \frac{\sum_{k=2}^{\infty}\left([k]_{q}-1\right)\left|a_{k} \| z\right|^{k-1}}{1-\sum_{k=2}^{\infty}\left|a_{k} \| z\right|^{k-1}} \leq \frac{\sum_{k=2}^{\infty}\left([k]_{q}-1\right)\left|a_{k}\right|}{1-\sum_{k=2}^{\infty}\left|a_{k}\right|} .
\end{aligned}
$$

This last expression is bounded above by $1-\beta$ if

which is equivalent to

$$
\sum_{k=2}^{\infty}\left([k]_{q}-1\right)\left|a_{k}\right| \leq(1-\beta)\left(1-\sum_{k=2}^{\infty}\left|a_{k}\right|\right),
$$

$$
\sum_{k=2}^{\infty}\left([k]_{q}-\beta\right) \mid a_{k} \leq 1-\beta .
$$

But (2.2) is true by hypothesis. Hence $\left|\frac{z \partial_{q} f(z)}{f(z)}-1\right| \leq 1-\beta$. This completes the proof.

As $q \rightarrow 1$, we have following result proved by Herb silverman[5].

Corollary 2.2. Let $f(z)=z+\sum_{k=2}^{\infty} a_{k} z^{k}$. if $\sum_{k=2}^{\infty}(k-\beta)\left|a_{k}\right| \leq 1-\beta$, then $f \in S^{*}(\beta)$.

As $q \rightarrow 1$ and $\beta=0$, we have following result, proved by Goodman [1].

Corollary 2.3. Let $f(z)=z+\sum_{k=2}^{\infty} a_{k} z^{k}$. if $\sum_{k=2}^{\infty} k \mid a_{k} \leq 1$, then $f \in S^{*}$.

Also special case of Theorem 2.1 when $q \rightarrow 1$ and $\beta=\frac{1}{2}$ proved by Schild [3].

Theorem 2.4. A function $f(z)=z+\sum_{k=2}^{\infty} a_{k} z^{k}$ is in $C_{q}(\beta)$, if

$$
\sum_{k=2}^{\infty}[k]_{q}\left([k]_{q}-\beta\right)\left|a_{k}\right| \leq 1-\beta .
$$

Proof: It can be easily seen that $f(z) \in C_{q}(\beta)$ if and only if $z \partial_{q} f(z) \in S_{q}^{*}(\beta)$. Since $z \partial_{q} f(z)=z+\sum_{k=2}^{\infty}[k]_{q} a_{k} z^{k}$, we may replace $a_{k}$ with $[k]_{q} a_{k}$ in the theorem.

As $q \rightarrow 1$, we have following result proved by Herb silverman[5]. 
Hamid Shamsan and S. Latha

Corollary 2.5. A function $f(z)=z+\sum_{k=2}^{\infty} a_{k} z^{k}$ is in $C(\beta)$, if $\sum_{k=2}^{\infty} k(k-\beta)\left|a_{k}\right| \leq 1-\beta$.

Theorem 2.6. A function $f(z)=z-\sum_{k=2}^{\infty}\left|a_{k}\right| z^{k}$ is in $T_{q}^{*}(\beta)$, if and only if

$$
\sum_{k=2}^{\infty}\left([k]_{q}-\beta\right)\left|a_{k}\right| \leq 1-\beta \text {. }
$$

Proof: In view of Theorem 2.1, it suffices to show the only if part. Assume that

$$
\operatorname{Re}\left\{\frac{z \partial_{q} f(z)}{f(z)}\right\}=\operatorname{Re}\left\{\frac{z-\sum_{k=2}^{\infty}[k]_{q}\left|a_{k}\right| z^{k}}{z-\sum_{k=2}^{\infty}\left|a_{k}\right| z^{k}}\right\}>\beta, \quad|z|<1 .
$$

Choose values of $z$ on the real axis so that $\frac{z \partial_{q} f(z)}{f(z)}$ is real. Upon clearing the denominator in (2.5) and letting $z \rightarrow 1$ through real values, we obtain

$$
1-\sum_{k=2}^{\infty}[k]_{q}\left|a_{k}\right| \geq \beta\left(1-\sum_{k=2}^{\infty}\left|a_{k}\right|\right) .
$$

Thus $\sum_{k=2}^{\infty}\left([k]_{q}-\beta\right)\left|a_{k}\right| \leq 1-\beta$, and this completes the proof.

As $q \rightarrow 1$, we have following result proved by Herb silverman [5].

Corollary 2.7. A function $f(z)=z-\sum_{k=2}^{\infty}\left|a_{k}\right| z^{k}$ is in $T^{*}(\beta)$, if and only if $\sum_{k=2}^{\infty}(k-\beta)\left|a_{k}\right| \leq 1-\beta$.

Corollary 2.8. If $f \in T_{q}^{*}(\beta)$ then $\left|a_{k}\right| \leq \frac{1-\beta}{[k]_{q}-\beta}$, with equality only for functions of the form $f_{k}(z)=z-\frac{(1-\beta) z^{k}}{[k]_{q}-\beta}$.

Theorem 2.9. A function $f(z)=z-\sum_{k=2}^{\infty}\left|a_{k}\right| z^{k}$ is in $C_{q}(\beta)$, if and only if

$$
\sum_{k=2}^{\infty}[k]_{q}\left([k]_{q}-\beta\right)\left|a_{k}\right| \leq 1-\beta
$$

Proof: The proof follows as that of the Theorem 2.4.

As $q \rightarrow 1$, we have following result proved by Herb silverman[5]. 
Corollary 2.10. A function $f(z)=z-\sum_{k=2}^{\infty}\left|a_{k}\right| z^{k}$ is in $C(\beta)$, if and only if $\sum_{k=2}^{\infty} k(k-\beta)\left|a_{k}\right| \leq 1-\beta$.

Theorem 2.11. If $f \in T$, then $\sum_{k=2}^{\infty}[k]_{q}\left|a_{k}\right| \leq 1$.

Proof: Suppose $\sum_{k=2}^{\infty}[k]_{q}\left|a_{k}\right|=1+\varepsilon,(\varepsilon>0)$. Then there exists an integer $N$ such that $\sum_{k=2}^{N}[k]_{q}\left|a_{k}\right|>1+\frac{\varepsilon}{2}$. For $z$ in the interval $\left(\frac{1}{1+\frac{\varepsilon}{2}}\right)^{\frac{1}{N-1}}<z<1$, we have
$\partial_{q} f(z) \leq 1-\sum_{k=2}^{N}[k]_{q}\left|a_{k}\right| z^{k-1} \leq 1-z^{N-1} \sum_{k=2}^{N}[k]_{q}\left|a_{k}\right|<1-\left(1+\frac{\varepsilon}{2}\right) z^{N-1}<0$. since $\partial_{q} f(0)>0$, there exists a real number $z_{0}, 0<z_{0}<1$, for which $\partial_{q} f\left(z_{0}\right)=0$. Hence $f(z) \notin T$, and the theorem is proved.

As $q \rightarrow 1$, we have following result proved by Herb silverman [5].

Corollary 2.12. If $f \in T$, then $\sum_{k=2}^{\infty} k\left|a_{k}\right| \leq 1$.

Theorem 2.13. If $f \in T_{q}^{*}(\beta)$, then

$$
r-\frac{1-\beta}{[2]_{q}-\beta} r^{2} \leq|f(z)| \leq r+\frac{1-\beta}{[2]_{q}-\beta} r^{2} \quad(|z|=r),
$$

with equality for $f(z)=z-\frac{(1-\beta) z^{2}}{[2]_{q}-\beta}, \quad(z= \pm r)$.

Proof: Note that $\left([2]_{q}-\beta\right) \sum_{k=2}^{\infty}\left|a_{k}\right| \leq \sum_{k=2}^{\infty}\left([k]_{q}-\beta\right)\left|a_{k}\right| \leq 1-\beta$, this last inequality following from Theorem 2.6. Thus

$$
|f(z)| \leq r+\sum_{k=2}^{\infty}\left|a_{k}\right| r^{k} \leq r+r^{2} \sum_{k=2}^{\infty}\left|a_{k}\right| \leq r+\frac{1-\beta}{[2]_{q}-\beta} r^{2}
$$

Similarly,

$$
|f(z)| \geq r-\sum_{k=2}^{\infty}\left|a_{k}\right| r^{k} \geq r-r^{2} \sum_{k=2}^{\infty}\left|a_{k}\right| \geq r-\frac{1-\beta}{[2]_{q}-\beta} r^{2} .
$$

As $q \rightarrow 1$, we have following result proved by Herb silverman[5].

Corollary 2.14. If $f \in T^{*}(\beta)$, then 
Hamid Shamsan and S. Latha

$$
r-\frac{1-\beta}{2-\beta} r^{2} \leq|f(z)| \leq r+\frac{1-\beta}{2-\beta} r^{2} \quad(|z|=r),
$$

with equality for $f(z)=z-\frac{(1-\beta) z^{2}}{2-\beta}, \quad(z= \pm r)$.

Theorem 2.15. If $f \in C_{q}(\beta)$, then

$$
r-\frac{1-\beta}{[2]_{q}\left([2]_{q}-\beta\right)} r^{2} \leq|f(z)| \leq r+\frac{1-\beta}{[2]_{q}\left([2]_{q}-\beta\right)} r^{2} \quad(|z|=r),
$$

with equality for $f(z)=z-\frac{(1-\beta) z^{2}}{[2]_{q}\left([2]_{q}-\beta\right)}, \quad(z= \pm r)$.

As $q \rightarrow 1$, we have following result proved by Herb silverman[5].

Corollary 2.16. If $f \in C(\beta)$, then

$$
r-\frac{1-\beta}{2(2-\beta)} r^{2} \leq|f(z)| \leq r+\frac{1-\beta}{2(2-\beta)} r^{2} \quad(|z|=r),
$$

with equality for $f(z)=z-\frac{(1-\beta) z^{2}}{2(2-\beta)}, \quad(z= \pm r)$.

Theorem 2.17. The disk $|z|<1$ is mapped onto a domain that contains the disk $|\omega|<\frac{[2]_{q}-1}{[2]_{q}-\beta}$ by any $f \in T_{q}^{*}(\beta)$, and onto a domain that contains the disk $|\omega|<\frac{\left.\left([2]_{q}-1\right)\left[[2]_{q}+1\right)-\beta\right]}{[2]_{q}\left([2]_{q}-\beta\right)}$, by any $f \in C_{q}(\beta)$. The theorem is sharp, with extremal functions $f(z)=z-\frac{(1-\beta) z^{2}}{[2]_{q}-\beta} \in T_{q}^{*}(\beta)$ and $f(z)=z-\frac{(1-\beta) z^{2}}{[2]_{q}\left([2]_{q}-\beta\right)} \in C_{q}(\beta)$.

Proof: The results follow upon letting $r \rightarrow 1$, in Theorem 2.13 and Theorem 2.15. As $q \rightarrow 1$, we get Theorem 5 in [5].

Theorem 2.18. If $f \in T_{q}^{*}(\beta)$, then

$$
1-\frac{[2]_{q}(1-\beta)}{[2]_{q}-\beta} r \leq\left|\partial_{q} f(z)\right| \leq 1+\frac{[2]_{q}(1-\beta)}{[2]_{q}-\beta} r \quad(|z|=r) .
$$

Equality holds for $f(z)=z-\frac{(1-\beta) z^{2}}{[2]_{q}-\beta}, \quad(z= \pm r)$.

Proof: We have 
Hamid Shamsan and S. Latha

$$
\left|\partial_{q} f(z)\right| \leq 1+\sum_{k=2}^{\infty}[k]_{q}\left|a_{k} \| z\right|^{k-1} \leq 1+r \sum_{k=2}^{\infty}[k]_{q}\left|a_{k}\right| .
$$

From Theorem 2.6, we have

$$
\sum_{k=2}^{\infty}[k]_{q}\left|a_{k}\right| \leq 1-\beta+\beta \sum_{k=2}^{\infty}\left|a_{k}\right| \leq 1-\beta+\frac{\beta(1-\beta))}{[2]_{q}-\beta}=\frac{[2]_{q}(1-\beta)}{[2]_{q}-\beta} \text {. }
$$

A substitution of (2.8) into (2.7) we obtained $\left|\partial_{q} f(z)\right| \leq 1+\frac{[2]_{q}(1-\beta)}{[2]_{q}-\beta} r$. On the other hand,

$$
\left|\partial_{q} f(z)\right| \geq 1-\sum_{k=2}^{\infty}[k]_{q}\left|a_{k} \| z\right|^{k-1} \geq 1-r \sum_{k=2}^{\infty}[k]_{q}\left|a_{k}\right| \geq 1-\frac{[2]_{q}(1-\beta)}{[2]_{q}-\beta} r,
$$

and the proof is complete.

As $q \rightarrow 1$, we have following result proved by Herb silverman[5].

Corollary 2.19. If $f \in T(\beta)$, then

$$
1-\frac{2(1-\beta)}{2-\beta} r \leq\left|f^{\prime}(z)\right| \leq 1+\frac{2(1-\beta)}{2-\beta} r \quad(|z|=r) .
$$

Equality holds for $f(z)=z-\frac{(1-\beta) z^{2}}{2-\beta}, \quad(z= \pm r)$.

Theorem 2.20. If $f \in C_{q}(\beta)$, then

$$
1-\frac{(1-\beta)}{[2]_{q}-\beta} r \leq\left|\partial_{q} f(z)\right| \leq 1+\frac{(1-\beta)}{[2]_{q}-\beta} r \quad(|z|=r) .
$$

Equality holds for $f(z)=z-\frac{(1-\beta) z^{2}}{[2]_{q}\left([2]_{q}-\beta\right)}, \quad(z= \pm r)$.

Theorem 2.21. If $f \in T_{q}^{*}(\beta)$, then $f$ is $q$-convex in the disk

$$
|z|<r(\beta)=\inf _{k}\left(\frac{[k]_{q}-\beta}{[k]_{q}^{2}(1-\beta)}\right)^{\frac{1}{k-1}} \quad(n=2,3, \ldots) .
$$

The result is sharp, with the extremal function being of the form $f_{k}(z)=z-\frac{(1-\beta) z^{k}}{(k-\beta)}$ for some $n$.

Proof: It suffices to show that $\left|\frac{z \partial_{q}^{2} f(z)}{\partial_{q} f(z)}\right| \leq 1$ for $|z| \leq r(\beta)$. We have 
Hamid Shamsan and S. Latha

$\left|\frac{z \partial_{q}^{2} f(z)}{\partial_{q} f(z)}\right|=\left|\frac{\sum_{k=2}^{\infty}[k]_{q}\left([k]_{q}-1\right)\left|a_{k}\right| z^{k-1}}{1+\sum_{k=2}^{\infty}[k]_{q}\left|a_{k}\right| z^{k-1}}\right| \leq \frac{\sum_{k=2}^{\infty}[k]_{q}\left([k]_{q}-1\right)\left|a_{k} \| z\right|^{k-1}}{1-\sum_{k=2}^{\infty}[k]_{q}\left|a_{k} \| z\right|^{k-1}}$.

Thus $\left|\frac{z \partial_{q}^{2} f(z)}{\partial_{q} f(z)}\right| \leq 1$ if $\quad \sum_{k=2}^{\infty}[k]_{q}\left([k]_{q}-1\right)\left|a_{k}\left\|\left.z\right|^{k-1} \leq 1-\sum_{k=2}^{\infty}[k]_{q}\left|a_{k} \| z\right|^{k-1}\right.\right.$,

or

$$
\sum_{k=2}^{\infty}[k]_{q}^{2}\left|a_{k} \| z\right|^{k-1} \leq 1
$$

According to Theorem 2.6, $\frac{\sum_{k=2}^{\infty}\left([k]_{q}-\beta\right)\left|a_{k}\right|}{(1-\beta)} \leq 1$. Hence (2.9) will be true if

$$
[k]_{q}^{2}|z|^{k-1} \leq \frac{[k]_{q}-\beta}{1-\beta} \quad(n=2,3, \ldots)
$$

Solving (2.10) for $z$, we obtain

$$
|z| \leq\left(\frac{[k]_{q}-\beta}{[k]_{q}^{2}(1-\beta)}\right)^{\frac{1}{k-1}} \quad(n=2,3, \ldots)
$$

Sitting $|z|=r(\beta)$ in (2.11), the result is follows.

Theorem 2.22. Let $f_{1}(z)=z$ and $f_{k}(z)=z-\frac{1-\beta}{[k]_{q}-\beta} z^{k} \quad(k=2,3, \ldots)$.

Then $f \in T_{q}^{*}(\beta)$ if and only if it can expressed in the form

$$
f(z)=\sum_{k=1}^{\infty} \lambda_{k} f_{k}(z), \text { where } \lambda_{k}>0 \text { and } \sum_{k=1}^{\infty} \lambda_{k}=1 .
$$

Proof: Suppose that

$$
\begin{aligned}
f(z) & =\sum_{k=1}^{\infty} \lambda_{k} f_{k}(z)=f_{1}(z)+\sum_{k=2}^{\infty} \lambda_{k} f_{k}(z)=\lambda_{1} z+\sum_{k=2}^{\infty} \lambda_{k}\left(z-\frac{1-\beta}{[k]_{q}-\beta} z^{k}\right) \\
& =\lambda_{1} z+\sum_{k=2}^{\infty} \lambda_{k} z-\sum_{k=2}^{\infty} \lambda_{k} \frac{1-\beta}{[k]_{q}-\beta} z^{k}=\left(\sum_{k=1}^{\infty} \lambda_{k}\right) z-\sum_{k=2}^{\infty} \lambda_{k} \frac{1-\beta}{[k]_{q}-\beta} z^{k} \\
& =z-\sum_{k=2}^{\infty} \lambda_{k} \frac{1-\beta}{[k]_{q}-\beta} z^{k} .
\end{aligned}
$$

Then

$$
\sum_{k=2}^{\infty} \lambda_{k}\left(\frac{1-\beta}{[k]_{q}-\beta}\right)\left(\frac{[k]_{q}-\beta}{1-\beta}\right)=\sum_{k=2}^{\infty} \lambda_{k}=1-\lambda_{1} \leq 1
$$


Thus $f \in T_{q}^{*}(\beta)$.

Conversely, suppose $f \in T_{q}^{*}(\beta)$. Since $\left|a_{k}\right| \leq \frac{1-\beta}{[k]_{q}-\beta}(k=2,3, \ldots)$, we may set $\lambda_{k}=\frac{[k]_{q}-\beta}{1-\beta}$ and $\lambda_{1}=1-\sum_{k=2}^{\infty} \lambda_{k}$. Then

$$
f(z)=\sum_{k=1}^{\infty} \lambda_{k} f_{k}(z)
$$

And the proof of Theorem 2.22 is complete.

As $q \rightarrow 1$, we have following result proved by Herb silverman[5].

Corollary 2.23. Let $f_{1}(z)=z$ and $f_{k}(z)=z-\frac{1-\beta}{k-\beta} z^{k} \quad(k=2,3, \ldots)$. Then $f \in T^{*}(\beta)$ if and only if it can expressed in the form $f(z)=\sum_{k=1}^{\infty} \lambda_{k} f_{k}(z)$,

where $\lambda_{k}>0$ and $\sum_{k=1}^{\infty} \lambda_{k}=1$.

\section{Partial sums}

In this section, we will examine the ratio of a function of the form (1.1) to its sequence of partial sums $f_{k}(z)=z+\sum_{n=2}^{k} a_{n} z^{n}$ when the coefficients of $f$ are sufficiently small to satisfy the condition (2.4). We will determine sharp lower bounds for $\Re\left\{\frac{f(z)}{f_{k}(z)}\right\}, \Re\left\{\frac{f_{k}(z)}{f(z)}\right\}, \Re\left\{\frac{\partial_{q} f(z)}{\partial_{q} f_{k}(z)}\right\}$ and $\mathfrak{R}\left\{\frac{\partial_{q} f_{k}(z)}{\partial_{q} f(z)}\right\}$.

Theorem 3.1. If $f$ of the form (1.1) and satisfies condition (2.4), then

$$
\mathfrak{R}\left\{\frac{f(z)}{f_{k}(z)}\right\} \geq 1-\frac{1}{c_{k+1}}, \quad(z \in \mathrm{U}, k \in \mathrm{N}),
$$

and

$$
\Re\left\{\frac{f_{k}(z)}{f(z)}\right\} \geq \frac{c_{k+1}}{1+c_{k+1}}, \quad(z \in \mathrm{U}, k \in \mathrm{N}),
$$

where $c_{k}=\frac{\left([k]_{q}-\beta\right)}{1-\beta}$. The estimates in (3.1) and (3.2) are sharp.

Proof: Suppose that $f$ satisfies condition (2.4), by Theorem 2.6, we have

$$
f \in T_{q}^{*}(\beta) \Leftrightarrow \sum_{n=2}^{\infty} c_{n}\left|a_{n}\right| \leq 1,
$$

It is easy to verify that $c_{n+1}>c_{n}>1$. Thus, 


\section{Hamid Shamsan and S. Latha}

We may write

$$
\sum_{n=2}^{k}\left|a_{n}\right|+c_{k+1} \sum_{n=k+1}^{\infty}\left|a_{n}\right| \leq \sum_{n=2}^{\infty} c_{n}\left|a_{n}\right| \leq 1 .
$$

$$
c_{k+1}\left\{\frac{f(z)}{f_{k}(z)}-\left(1-\frac{1}{c_{k+1}}\right)\right\}=\frac{1+\sum_{n=2}^{k} a_{n} z^{n-1}+c_{k+1} \sum_{n=k+1}^{\infty} a_{n} z^{n-1}}{1+\sum_{n=2}^{k} a_{n} z^{n-1}}=\frac{1+A(z)}{1+B(z)} .
$$

Set

$$
\frac{1+A(z)}{1+B(z)}=\frac{1+w(z)}{1-w(z)}
$$

so that $w(z)=\frac{B(z)-A(z)}{2+B(z)+A(z)}$, then $w(z)=\frac{c_{k+1} \sum_{n=k+1}^{\infty} a_{n} z^{n-1}}{2+2 \sum_{n=2}^{k} a_{n} z^{n-1}+c_{k+1} \sum_{n=k+1}^{\infty} a_{n} z^{n-1}}$,

and

$$
|w(z)| \leq \frac{c_{k+1} \sum_{n=k+1}^{\infty}\left|a_{n}\right|}{2-2 \sum_{n=2}^{k}\left|a_{n}\right|-c_{k+1} \sum_{n=k+1}^{\infty}\left|a_{n}\right|} .
$$

Now $|w(z)| \leq 1$ if and only if $\sum_{n=2}^{k}\left|a_{n}\right|+c_{k+1} \sum_{n=k+1}^{\infty}\left|a_{n}\right| \leq 1$,

which is true by (3.3). This readily yields the assertion (3.1).

To see that

$$
f(z)=z-\frac{z^{k+1}}{c_{k+1}}
$$

gives sharp results, we observe that for $z=r e^{\frac{\pi i}{k}}$

$$
\frac{f(z)}{f_{k}(z)}=1+\frac{z^{k}}{c_{k+1}}
$$

Letting $z \rightarrow 1^{-}$, we have $\frac{f(z)}{f_{k}(z)}=1-\frac{1}{c_{k+1}}$,

which shows that the bounds in (3.1) are the best possible for each $n \in \mathrm{N}$.

In the same way we take

$$
\left(1+c_{k+1}\right)\left(\frac{f_{k}(z)}{f(z)}-\frac{c_{k+1}}{1+c_{k+1}}\right)=\frac{1+\sum_{n=2}^{\infty} a_{n} z^{n-1}+c_{k+1} \sum_{n=k+1}^{\infty} a_{n} z^{n-1}}{1+\sum_{n=2}^{\infty} a_{n} z^{n-1}}=\frac{1+w(z)}{1-w(z)},
$$


Hamid Shamsan and S. Latha

where $\quad|w(z)| \leq \frac{1+c_{k+1} \sum_{n=k+1}^{\infty}\left|a_{n}\right|}{2-2 \sum_{n=2}^{\infty}\left|a_{n}\right|-\left(1+c_{k+1}\right) \sum_{n=k+1}^{\infty}\left|a_{n}\right|}$.

Now $|w(z)| \leq 1$ if and only if $\sum_{n=2}^{k}\left|a_{n}\right|+\left(1+c_{k+1}\right) \sum_{n=k+1}^{\infty}\left|a_{n}\right| \leq 1$,

which is true by (3.3). This readily yields the assertion (3.2).

The estimate in (3.2) is sharp with the extremal function $f(z)$ given by (3.4). This completes the proof of Theorem.

Theorem 3.2. If $f$ If $f$ of the form (1.1) and satisfies condition (2.4), then

$$
\Re\left\{\frac{\partial_{q} f(z)}{\partial_{q} f_{k}(z)}\right\} \geq 1-\frac{[k]_{q}+1}{c_{k+1}}, \quad(z \in \mathrm{U}, k \in \mathrm{N}),
$$

and

$$
\Re\left\{\frac{\partial_{q} f_{k}(z)}{\partial_{q} f(z)}\right\} \geq \frac{c_{k+1}}{[k]_{q}+1+c_{k+1}}, \quad(z \in \mathrm{U}, k \in \mathrm{N}) .
$$

where $c_{k}=\frac{\left([k]_{q}-\beta\right)}{1-\beta}$. The estimates in (3.5) and (3.6) are sharp with the extremal function given by (3.4).

Proof: We may write

$$
c_{k+1}\left\{\frac{\partial_{q} f(z)}{\partial_{q} f_{k}(z)}-\left(1-\frac{[k]_{q}+1}{c_{k+1}}\right)\right\}=\frac{1+\sum_{n=2}^{k}[n]_{q} a_{n} z^{n-1}+\frac{c_{k+1}}{[k]_{q}+1} \sum_{n=k+1}^{\infty}[n]_{q} a_{n} z^{n-1}}{1+\sum_{n=2}^{k}[n]_{q} a_{n} z^{n-1}}=\frac{1+A(z)}{1+B(z)} .
$$

Set $\frac{1+A(z)}{1+B(z)}=\frac{1+w(z)}{1-w(z)}$, so that $w(z)=\frac{B(z)-A(z)}{2+B(z)+A(z)}$$$
\text { then } w(z)=\frac{[k]_{q}+1}{2+2 \sum_{n=k+1}^{k}[n]_{q} a_{n} z^{n-1}+\frac{c_{k+1}}{[k]_{q}+1} \sum_{n=k+1}^{\infty}[n]_{q} a_{n} z^{n-1}}
$$

and

$$
|w(z)| \leq \frac{\frac{c_{k+1}}{[k]_{q}+1} \sum_{n=k+1}^{\infty}[n]_{q}\left|a_{n}\right|}{2-2 \sum_{n=2}^{k}[n]_{q}\left|a_{n}\right|-\frac{c_{k+1}}{[k]_{q}+1} \sum_{n=k+1}^{\infty}[n]_{q}\left|a_{n}\right|}
$$


Hamid Shamsan and S. Latha

Now $|w(z)| \leq 1$ if and only if $\frac{c_{k+1}}{[k]_{q}+1} \sum_{n=k+1}^{\infty}[n]_{q}\left|a_{n}\right|+\sum_{n=2}^{k}[n]_{q}\left|a_{n}\right| \leq 1$.

From the condition (2.4), it is suffices to show that

$$
\frac{c_{k+1}}{[k]_{q}+1} \sum_{n=k+1}^{\infty}[n]_{q}\left|a_{n}\right|+\sum_{n=2}^{k}[n]_{q}\left|a_{n}\right| \leq c_{n}\left|a_{n}\right| .
$$

This is equivalent to showing that

$$
\sum_{n=2}^{k}\left(c_{n}-[n]_{q}\right)\left|a_{n}\right|+\sum_{n=k+1}^{\infty} \frac{\left([k]_{q}+1\right) c_{n}-n c_{k+1}}{[k]_{q}+1} \geq 0 .
$$

To prove the second part of this theorem, we write

$$
\begin{aligned}
& w(z)=\left([k]_{q}+1+c_{k+1}\right)\left\{\frac{\partial_{q} f(z)}{\partial_{q} f_{k}(z)}-\left(1-\frac{[k]_{q}+1}{c_{k+1}}\right)\right\}=1-\frac{\left(1+\frac{c_{k+1}}{[k]_{q}+1}\right) \sum_{n=k+1}^{\infty}[n]_{q} a_{n} z^{n-1}}{1+\sum_{n=2}^{k}[n]_{q} a_{n} z^{n-1}} \\
& \text { yields }\left|\frac{w(z)-1}{w(z)+1}\right| \leq \frac{\left(1+\frac{c_{k+1}}{[k]_{q}+1}\right) \sum_{n=k+1}^{\infty}[n]_{q}\left|a_{n}\right|}{2-2 \sum_{n=2}^{k}[n]_{q}\left|a_{n}\right|-\left(1+\frac{c_{k+1}}{[k]_{q}+1}\right) \sum_{n=k+1}^{\infty}[n]_{q}\left|a_{n}\right|} \leq 1,(z \in \mathcal{U}), \\
& \text { if and only if } 2\left(1+\frac{c_{k+1}}{[k]_{q}+1}\right) \sum_{n=k+1}^{\infty}[n]_{q}\left|a_{n}\right| \leq 2-2 \sum_{n=2}^{k}[n]_{q}\left|a_{n}\right| .
\end{aligned}
$$

The bound in (3.6)is sharp for all $n \in \mathrm{N}$ with the extremal function (3.4). This completes the proof.

\section{REFERENCES}

1. A.W.Goodman, Univalent functions and nonanalytic curves, Poroc. Amer. Math. Soc., 8(3)(1957) 598-601.

2. F.H.Jackson, On $q$-functions and a certain difference operator, Trans. Royal Soc. Edinburgh, 46 (1909) 253-281.

3. A.Schild, On a class of univalent, star shaped mappings, Poroc. Amer. Math. Soc., 9(5) (1958) 751-757.

4. T.M.Seoudy and M.K.Aouf, Coefficient estimates of new classes of q-starlike and qconvex functions of complex order, J. Math. Inequal, 10(1) (2016) 135-145.

5. H.Silverman, Univalent functions with negative coefficients, Poroc. Amer. Math. Soc., 51(1) (1975) 109-116.

6. H.Silverman, Partial sums of starlike and convex functions, J. Math. Anal. Appl., 209(1) (1997) 221-227.

7. H.Shamsan, F.Alsarari and S.Latha, On coefficients inequalities of functions related to $q$-derivative and conic regions, J.Math. Informatics, 9 (2017) 9-17. 